\title{
Atherosclerotic Diseases Championing Death Toll: Instituting Pediatric Hyperlipidemia Screening Programme
}

\author{
Sikandar Hayat Khan \\ Department of Pathology, Naval Hospital, Islamabad, Pakistan
}

Atherosclerotic cardiovascular diseases (ASCVD), undeniably top any available list of mortality and morbidity statistics charts all over the globe. One in 4 deaths with every death/36 seconds has been attributed to ASCVD in United States. ${ }^{1}$ The study of various animal models have already established that the process of atherosclerosis starts from early childhood and the atherosclerotic plaque formation is highly dependent upon multiple genetic and epigenetic factors. ${ }^{2,3}$ The lifestyle risks associated with hyperlipidemia include both the dietary habits and sedentary lifestyles.

Within lastfew decades, scientific discoveries have allowed valuable insights into moleculardefects leading to aspecific hyperlipidemia phenotype. These hyperlipidemia patterns include hypercholesterolemia, hypertriglyceridemia, combined hyperlipidemia and low HDL and LDL cholesterol categories. From genetics point of view, the disease can be categorised into monogenic disorders like familial hypercholesterolemia (FH) and polygenic disorders where additive effects of multiple single nucleotide phenotypes (SNPs). ${ }^{4}$ There are novel sequencing methods like next-generation sequencing (NGS) and exom sequencing (EB) which leads to ASCVD the exact molecular defects; however, cost, equipment and expertise are various limitations for routine clinical use. Literature review clearly highlights the ever increasing findings of hyperlipidemia in pediatric age group. ${ }^{5}$ While molecular pathology diagnosis may not become accessible in clinical arena, there still remains an urgent need to preempt lipid disorders as early as possible to avoid future ASCVD issues in productive age group, especially in developing world.

The current practice in developing and certain developed countries does not cater for screening pediatric population for hyperlipidemia. While hyperlipidemia, like $\mathrm{FH}$, remains one of the commonest genetic disorders and causative factor for ASCVDassociated complications like myocardial infarction, stroke and peripheral vascular disease.

Correspondence to: Dr. Sikandar Hayat Khan, Department of Pathology, Naval Hospital, Islamabad, Pakistan E-mail:sik_cpsp@yahoo.com

Received: October 03, 2020; Revised: October 20, 2020;

Accepted: October 26, 2020

DOI: https://doi.org/10.29271/jcpsp.2020.11.1119
Screening with lipid profile and treatment remain not only available but also cost-effective and efficient. ${ }^{6}$ Allowing lipid to deposit within the plaques in earlier decades of life and then facing the disease burden over in young or in middle years of life is neither desirable nor avoids in loss of experienced manpower. Death by sudden cardiac arrest is no more an unknown entity with over 300,000 to 400,000 per annum succumbing to it in developed countries like United States. ${ }^{7}$ Similarly, strokes are also a major source of mortality and morbidity with multifactorial etiologies including hyperlipidemia. ${ }^{8}$

Recently, the recognition for early screening of hyperlipidemia in pediatric and adolescent population is emerging. The Slovenian model utilised a 2-step approach with total cholesterol screening followed by genetic testing for FH to identify 280 children with hypercholesterolemia, where genetic defects in LDL receptor (28.9\%), ApoB mutations in $15.9 \%$ and $40.9 \%$ having ApoE4 were identified. ${ }^{9}$ The timings related to pediatric screening are varying in literature. John et al. in line with American Association of Pediatrics (AAP) have advocated screening between 2 to 10 years of age with fasting lipid profile. ${ }^{10} \mathrm{However}$, the guidelines from US Preventive Services Task Force (USPSTF) on pediatric hyperlipidemia have recommended two age groups including 9 to 11 years and 16 to 19 years with higher diagnostic yield in children having higher body mass index. ${ }^{11}$ In contrast, Lozano et al. systematically reviewed the guidelines for USPSTF and recommended that lipid screening and onward therapeutic management has beneficial effects only in short-term. However, the author could not demonstrate long-term positive outcomes in subjects with $\mathrm{FH}^{6}{ }^{6}$ Provided compelling evidence, there remains a gap between incorporating such hyperlipidemia guidelines for screening in the developing nations. Reasons could be multifold including resource availability, lack of governmental oversight, and possibly lack of emphasis on this primary care requirement. It can be seen that the major killers for most countries including South East Asian countries remain metabolic diseases including ischemic heart diseases (IHD), strokeand diabetes mellitus. ${ }^{1}$ People with underlying metabolic ailments are the most vulnerable category to acquire new and remerging infections including COVID-19. So, in all possiblecasescenario, itissuggested to incorporatehyperlipidemia screening at primary healthcare set up at least as per any of the above recommendations. 
Mass screening or criteria-based population-wide screening is recommended for disorders and diseases where a diagnostic approach is available, which must be cost-effective; and therapeutic modalities are easily accessible. The developing countries are losing productive work force, suffering economical loss and emotional trauma by sudden death among young family members. In case of hyperlipidemia, either resulting monogenic or polygenic lipid disorder or secondarily appearing as part of another metabolic disorder, it is suggested that a task force may be needed to address one of the most fatal group of diseases. The taskforce needs to define an appropriate timeline for screening by a simple total cholesterol, which may be further shortlisted by collecting appropriate family history. However, a simple cholesterol test may be carried out by $10 \pm 2$ years of age and a repetition may be considered in adolescence. Children and adolescents may further be narrowed down for both lifestyle changes and possible medicinal therapy. This practice, if adopted, will not only add years to life, but will boost quality of life in those extended years.

\section{REFERENCES}

1. Centre for disease control and prevention. Heart Disease in the United States. http://www.cdc.gov/heartdisease/docs/ ConsumerEd_HeartDisease.pdf. Accessed 27-Sep-2020.

2. Zhao Y, Yang Y, Xing R, Cui X, Xiao Y, Xie L, et al. Hyperlipidemia induces typical atherosclerosis development in Ldlr and Apoe deficient rats. Atherosclerosis 2018; 271: 26-35. doi: 10.1016/j.atherosclerosis.2018.02.015.

3. Kumar S, Kang DW, Rezvan A, Jo H. Accelerated atherosclerosis development in C57BI6 mice by overexpressing AAV-mediated PCSK9 and partial carotid ligation. Lab Invest 2017; 97(8):935-945. doi: 10.1038/ labinvest.2017.47.
4. Brown EE, Sturm AC, Cuchel M, Braun LT, Duell PB, Underberg JA, et al. Genetic testing in dyslipidemia: A scientific statement from the national lipid association. J Clin Lipidol 2020; 14(4):398-413. doi: 10.1016/j.jacl. 2020.04.011.

5. Opina AD, Cephus C, Wang Y, Younan S, Moodie D. The effects of lifestyle changes on serum lipid levels in children in a real life setting. Congenit Heart Dis 2018; 13(2):327-333. doi: 10.1111/chd.12580.

6. Lozano P, Henrikson NB, Dunn J, Morrison CC, Nguyen M, Blasi PR, et al. Lipid screening in childhood and adolescence for detection of familial hypercholesterolemia: Evidence report and systematic review for the us preventive services task force. JAMA 2016; 316(6):645-55. doi: 10.1001/jama.2016.6176. PMID: 27532919.

7. Kuriachan VP, Sumner GL, Mitchell LB. Sudden cardiac death. Curr Probl Cardiol 2015; 40(4):133-200. doi: 10.1016/j.cpcardiol.2015.01.002.

8. Guzik A, Bushnell C. Stroke epidemiology and risk factor management. Continuum (minneap minn) 2017; 23(1): 15-39. doi: 10.1212/CON.0000000000000416.

9. Groselj U, Kovac J, Sustar U, Mlinaric M, Fras Z, Podkrajsek $\mathrm{KT}$, et al. Universal screening for familial hypercholesterolemia in children: The slovenian model and literature review. Atherosclerosis 2018; 277:383-91. doi: 10.1016/j.atherosclerosis.2018.06.858.

10. John C, Neal W. Screening children for hyperlipidemia by primary care physicians in West Virginia. W V Med J 2012; 108(3):30-5.

11. Lozano P, Henrikson NB, Morrison CC, Dunn J, Nguyen M, Blasi $P$, et al. Lipid screening in childhood for detection of multifactorial dyslipidemia: A systematic evidence review for the U.S. preventive services task force. Rockville (MD): Agency For Healthcare Research And Quality (US) 2016; Report No. 14-05204-EF-1. PMID: 27559550. 